\title{
CORRESPONDENCE OPEN \\ Elevated serum levels of S100A8/A9 and HMGB1 at hospital admission are correlated with inferior clinical outcomes in COVID-19 patients
}

\author{
Liting Chen ${ }^{1}$, Xiaolu Long ${ }^{1}$, Qian Xu ${ }^{1}$, Jiaqi Tan ${ }^{2}$, Gaoxiang Wang ${ }^{1}$, Yang Cao, Jia Wei ${ }^{1}$, Hui Luo ${ }^{1}$, Hui Zhu ${ }^{3}$, Liang Huang ${ }^{1}$, Fankai Meng ${ }^{1}$, \\ Lifang Huang ${ }^{1}$, Na Wang ${ }^{1}$, Xiaoxi Zhou ${ }^{1}$, Lei Zhao ${ }^{1}$, Xing Chen ${ }^{1}$, Zekai Mao ${ }^{1}$, Caixia Chen ${ }^{1}$, Zhen Li ${ }^{3}$, Ziyong Sun ${ }^{4}$, Jianping Zhao ${ }^{5}$, \\ Daowen Wang ${ }^{6}$, Gang Huang ${ }^{7}$, Wei Wang ${ }^{8}$ and Jianfeng Zhou ${ }^{1}$
}

Cellular \& Molecular Immunology (2020) 17:992-994; https://doi.org/10.1038/s41423-020-0492-x

COVID-19 is a disease with heterogeneous clinical appearances. Most patients are asymptomatic or exhibit mild to moderate symptoms; approximately $15 \%$ progress to severe pneumonia and about $5 \%$ are eventually admitted to the intensive care unit (ICU) due to acute respiratory distress syndrome (ARDS), septic shock and/ or multiple organ failure. ICU patients respond poorly to currently available treatments and exhibit a high mortality rate. ${ }^{1-3}$ Inadequate identification of the determinants of fatal outcomes is one of the major obstacles to the improvement of the outcomes in severe COVID-19 patients. A previous study reported a scoring system (COVID-GRAM) which accurately predicted the occurrence of critical illness in hospitalized COVID-19 patients. ${ }^{4}$ Damage-associated molecular patterns (DAMPs), or alarmins, are a number of molecules, released by stressed cells undergoing microbial infection or sterile injury, that act as danger signals to promote and exacerbate the inflammatory response. ${ }^{5,6}$ Of note, the serum level of S100A8/A9 and HMGB1 was found to be correlated with both the severity of pathogen-associated tissue damage and excessive cytokine storm. ${ }^{7}$ Despite the hypothesis that S100A8/A9 and HMGB1 are significantly involved in COVID-19, so far, no study has yet tried to substantiate the hypothesis. In this study, we aimed to define the role of S100A8/ A9 and HMGB1 in progression to a fatal outcome and develop clinically relevant risk strata for COVID-19 patients.

A total of 121 patients were enrolled in this retrospective study, of which 40 patients were in ICU and 81 patients in general wards at enrollment (Table S1). ICU Patients had much higher COVID-GRAM risk scores in comparison to those in general wards. Complications, including ARDS, sepsis, septic shock, secondary infection, acute renal injury, acute cardiac injury or failure, were more frequent in CCOVID19 patients admitted to ICU. As of the cutoff date of April 30, 2020, most of non-ICU patients $(96.3 \%)$ had been discharged alive, while $82.5 \%$ of ICU patients had died in ICU.
COVID-19 patients treated in general wards had significantly elevated level of S100A8/A9 $(P=0.033)$ but not HMGB1 $(P>0.9999)$ as compared to healthy controls, suggesting that S100A8/A9 is a more sensitive alarmin than HMGB1 in response to SARS-CoV-2 infection. However, both S100A8/A9 and HMGB1 were significantly elevated extracellularly in ICU-admission patients compared to nonICU patients, or in fatal outcomes patients compared to alive patients (Fig. 1a-d), indicating that significant elevation of S100A8/ A9 and HMGB1 was associated with high mortality.

We further examined the Spearman's correlation between the serum S100A8/A9 or HMGB1 levels and clinical manifestations in COVID-19 patients. First of all, either serum levels of S100A8/A9 or HMGB1 at admission were positively correlated with peak CT score and oxygen demand, which is indicative of the severity of acute lung injury and ARDS (Fig. 1e, f and S1a, b). Moreover, the degree of organic impairment, as evaluated by the MCP classification, NTproBNP level, cTn I level, and AKI stage were well correlated with the serum levels of S100A8/A9 or HMGB1 (Fig. S1c-j). The level of peak D-dimer significantly was elevated as the serum S100A8/A9 or HMGB1 increased (Fig. $1 \mathrm{~g}, \mathrm{~h}$ ). On the other hand, the ratio of neutrophils to lymphocytes was positively correlated with the serum S100A8/A9 but not with HMGB1, suggesting S100A8/A9 plays a more important role in the substantial reduction of the peripheral lymphocytes. The serum S100A8/A9 was strongly correlated with the qSOFA score, ${ }^{8}$ a quick indicator of sepsisrelated organ dysfunction, indicating that patients with higher S100A8/A9 or HMGB1 levels tended to suffer from more severe sepsis-related organ dysfunction (both $P<0.0001$ ) (Fig. 1i-l).

We then evaluated the potential correlation between levels of S100A8/A9 or HMGB1 and COVID-GRAM risk scores. Either levels of S100A8/A9 $(P<0.0001)$ or HMGB1 $(P=0.030)$ significantly correlated with the COVID-GRAM risk scores (Fig. 1m, n). Serum levels of S100A8/

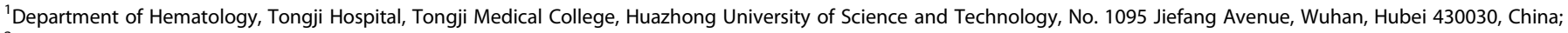

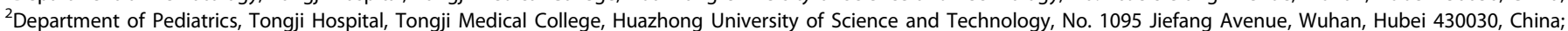

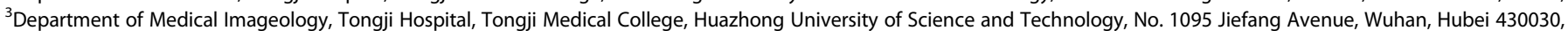

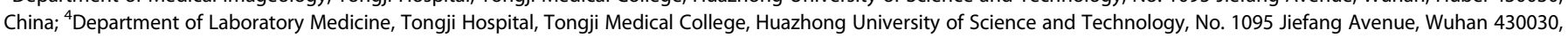

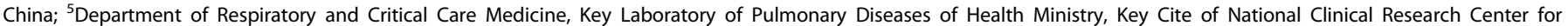

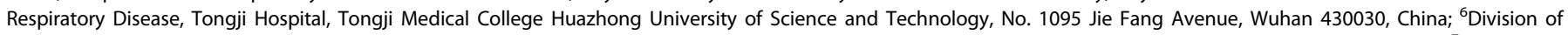

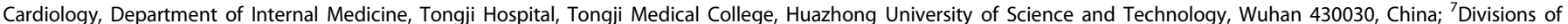

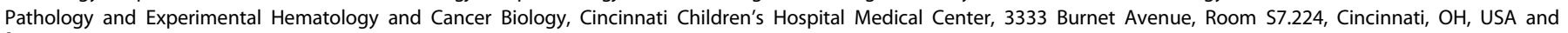
${ }^{8}$ Department of Neurology, Tongji Hospital, Tongji Medical College, Huazhong University of Science and Technology, No. 1095 Jiefang Avenue, Wuhan 430030 Hubei, China Correspondence: Gang Huang (Gang.Huang@cchmc.org) or Wei Wang (wwang@vip.126.com) or Jianfeng Zhou (jfzhou@tjh.tjmu.edu.cn)

These authors contributed equally: Liting Chen, Xiaolu Long, Qian Xu, Jiaqi Tan
}

Received: 14 June 2020 Accepted: 18 June 2020

Published online: 3 July 2020 
a

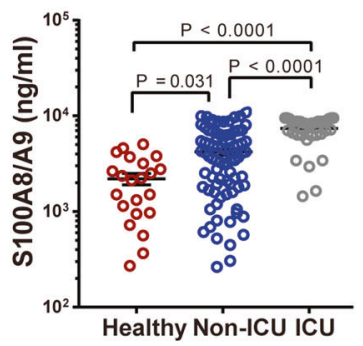

e

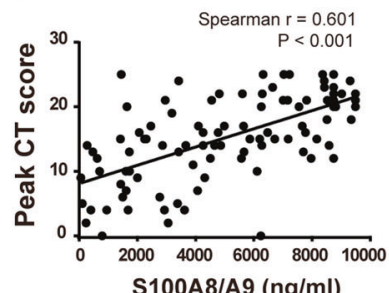

S100A8/A9 (ng/ml)

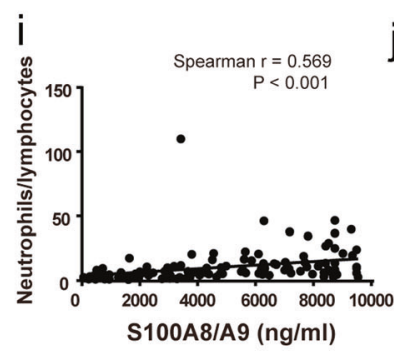

m

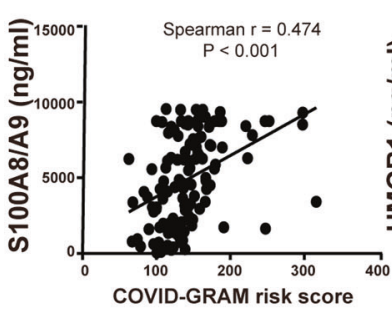

$\mathrm{n}$

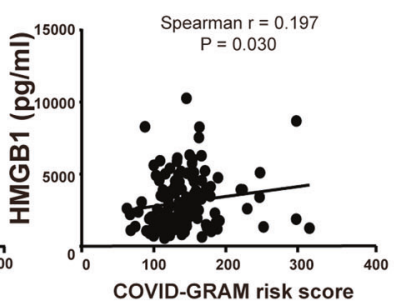

C

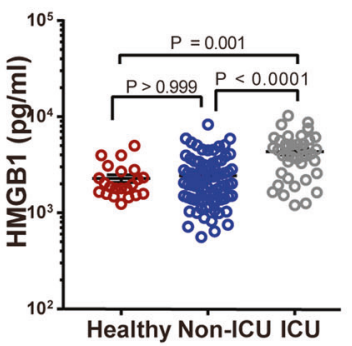

f
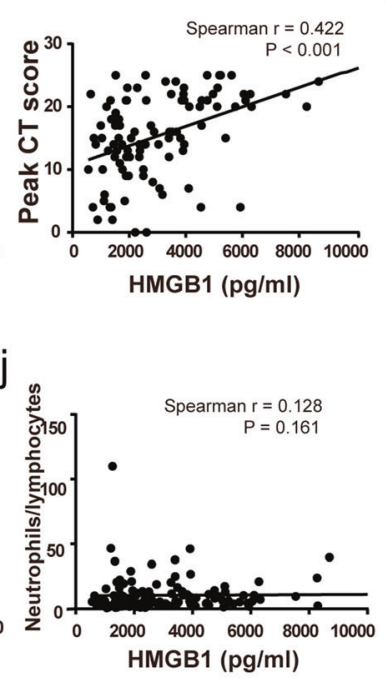

HMGB1 (pg/ml)

g
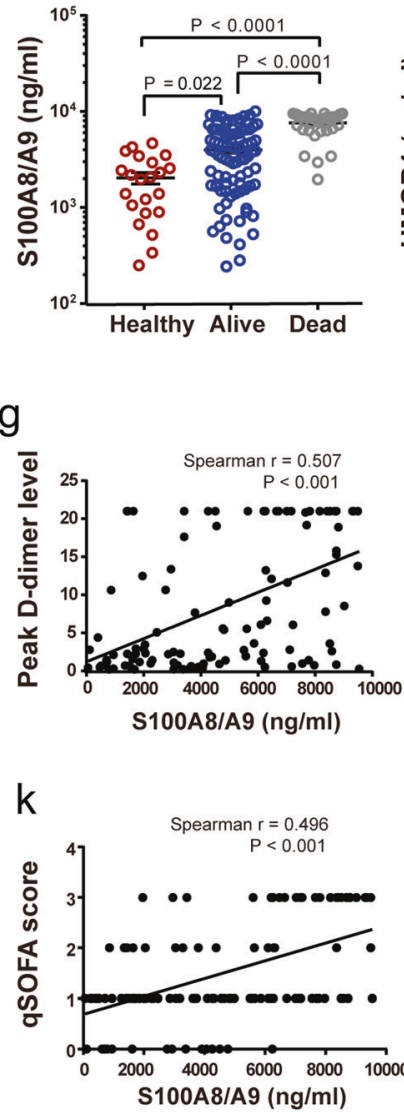

0
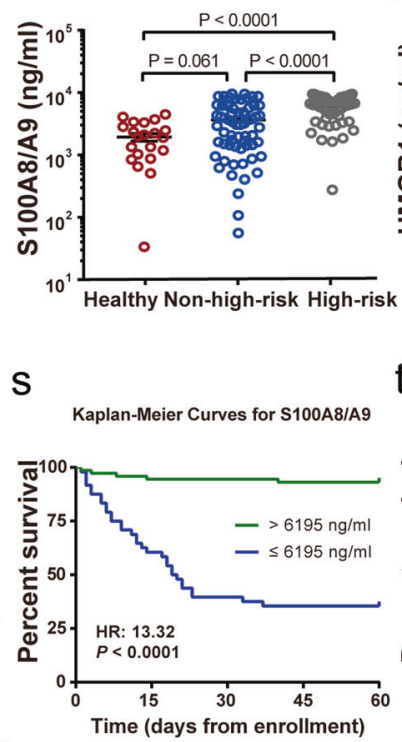

$\mathrm{p}$

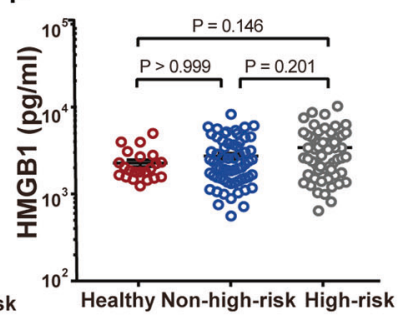

$\mathrm{t}$

d

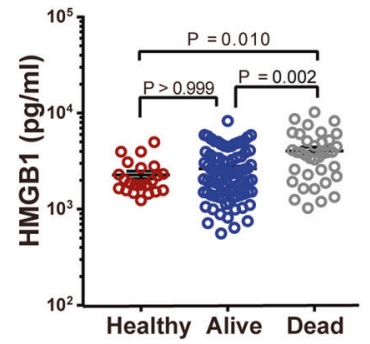

h
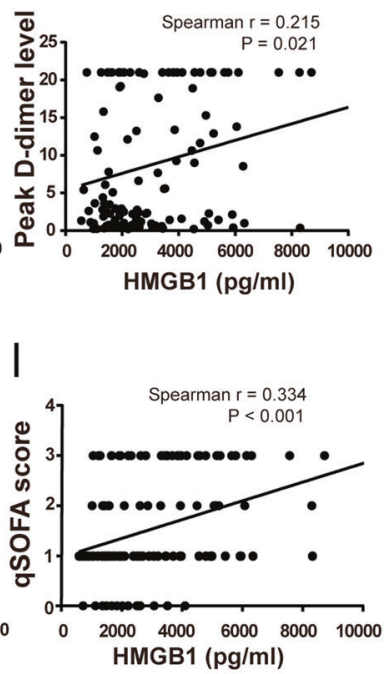

Kaplan-Meier Curves for COVID-GRAM

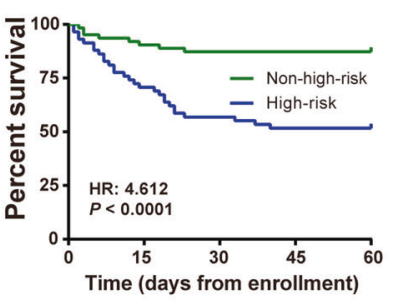

Fig. 1 Serum levels of S100A8/A9 and HMGB1 were strongly correlated with the severity of clinical manifestations and with great predictive power for the risk for ICU admission and death. a, b Comparison of S100A8/A9 and HMGB1 levels between healthy people, non-ICU patients, and ICU patients of COVID-19. c, d Comparison of S100A8/A9 and HMGB1 levels between healthy people, alive patients and dead patients of COVID-19. Spearman's correlation analyses between S100A8/A9 or HMGB1 levels and peak CT score (e, f), D-dimer level (g, h), neutrophil/ lymphocyte ratio (i, j), and quick Sequential Organ Failure Assessment (qSOFA) scores (k, I). $\mathbf{m}, \mathbf{n}$ Spearman's correlation analyses of S100A8/A9 or HMGB1 and COVID-GRAM risk score. o, p Comparison of S100A8/A9 or HMGB1 between healthy people and COVID-19 patients of different risk groups (divided according to COVID-GRAM risk score). $\mathbf{q}, \mathbf{r}$ Receiver operating characteristic (ROC) curve evaluation of the performance of S100A8/A9, HMGB1, combined S100A8/A9 and HMGB1, and COVID-GRAM risk score in distinguish COVID-19 patients with ICU admission or subsequent death. Sixty days survival is shown for patients with different circulating S100A8/A9 levels (s) and different COVID-GRAM risks (t) by Kaplan-Meier curves 
A9 but not HMGB1 were significantly increased in patient with high risk of COVID-GRAM risk scores which were defined according to the online calculator (http://118.126.104.170) ${ }^{4}$ (Fig. 1o, p), highlighting that $\mathrm{S100A8/A9}$ is a better prognostic indicator than HMGB1.

The prognostic values of S100A8/A9, HMGB1 or COVID-GRAM risk scores were further evaluated by ROC analysis and their areas under curve were calculated (Fig. 1q, r). For the prediction of ICU admission, the AUCs for COVID-GRAM risk scores with S100A8/A9, HMGB1 and S100A8/A9 and HMGB1 in combination were 0.810, $0.860,0.781$ and 0.901 , respectively (Table S2). For the prediction of subsequent death, the AUCs for COVID-GRAM risk scores, S100A8/A9, HMGB1 and S100A8/A9 and HMGB1 in combination were $0.818,0.875,0.694$ and 0.881 , respectively (Table S2). The sensitivity and specificity of S100A8/A9 and HMGB1 of the ROC curves illustrated in Fig. 1 $q, r$ were listed in Table S2. The combination of S100A8/A9 with HMGB1 increased the prediction power compared to $\mathrm{S100A8/A9}$ or HMGB1 alone although no significant differences were observed statistically (Table S2).

Moreover, higher S100A8/A9 level $(P<0.0001)$ or higher COVIDGRAM risk score $(P<0.0001)$ resulted in significant worse overall survival (Fig. 1s, t). The COVID-19 patients were classified into low or high level groups according to the concentrations of S100A8/ A9 at a cutoff of $6195 \mathrm{ng} / \mathrm{ml}$, which was set by maximizing Youden's index according to the ROC curves (Fig. 1s). Meanwhile, the COVID-19 patients were also classified into high-risk or nonhigh-risk groups according to COVID-GRAM risk scores (Fig. 1t). The hazard ratio of high S100A8/A9 level was 13.32, which was greater than that of COVID-GRAM risk score $(H R=4.612)$. The concentrations of S100A8/A9 measured at hospital admission showed better predictive power than COVID-GRAM risk scores for subsequent death in COVID-19 patients.

To explore the possible correlation between S100A8/A9 or HMGB1 and cytokine storm in COVID-19, the serum levels of S100A8/A9 or HMGB1 were analyzed by Spearman's correlation tests to know their correlation with of each of the 48 cytokine concentrations in individual patients (Table S3). While the serum levels of S100A8/A9 or HMGB1 correlated with the concentrations of a different spectrum of pro-inflammatory cytokines, 12

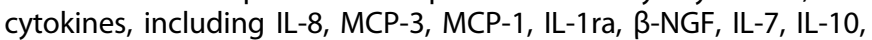
RANTES, G-CSF, IL-1a, CTACK and IL-17A, simultaneously correlated with both the S100A8/A9 and HMGB1. Interestingly, 3 myeloid chemokines, IL-8, MCP-3 and MCP-1, were among the most significant cytokines simultaneously correlated with both the S100A8/A9 and HMGB1 and showed the lowest $P$ value $(P<$ 0.0001 ), indicating that the overproduced S100A8/A9 and HMGB1 in serum were associated with distinct signatures for cytokine storm in patients with COVID-19. ${ }^{9,10}$

In conclusion, this study identified two alarmins, especially S100A8/A9, could accurately identify patients who were subsequently admitted to ICU wards or died with a predictive precision similar to or better than COVID-GRAM risk score. Taking S100A8/ A9 as a predictor for COVID-19 might offer some advantages over other clinical or laboratory indicators, since it is easy to measure and the result is easy to interpret. As a single parameter alone, to our knowledge, S100A8/S100A9 is the only one helpful for an early identification of COVID-19 patients who may be admitted to ICU admission or facing death. Using S100A8/A9 to distinguish COVID19 patients with fatal outcomes is of great clinical significance.

\section{ACKNOWLEDGEMENTS}

We appreciate eStart Medical Technology. CO., Ltd., Professor \& Dr. Jie Hou, Meng Chen, Zhongyan Zhang, Xu Ji, Chun Li, etc. for discussion of study design, medical affairs, data management, and statistics. We also appreciate Professor Guangliang Shan, Wei Han, PhD, from the Department of Epidemiology and Biostatistics, School of Basic Medicine, Peking Union Medical College, for their kind assistance in statistical analysis. This work was supported by the Emergency Research Project of Tongji hospital (to Dr. JZ), Emergency Research Project of Tongji hospital of Huazhong University of Science and Technology (2020kfyXGYJ045, to Dr. JZ), Emergency Research Project of Hubei province (2020FCA006, to Dr. WW).

\section{AUTHOR CONTRIBUTIONS}

Conception and design: Jianfeng $\mathrm{Z}, \mathrm{WW}$ and $\mathrm{GH}$. Acquisition, analysis, or interpretation of data: $\mathrm{LC}, \mathrm{XL}, \mathrm{QX}, \mathrm{JT}, \mathrm{GW}, \mathrm{YC}, \mathrm{JW}, \mathrm{HL}, \mathrm{HZ}, \mathrm{CC}$. Patient care and clinical information provision: $Y C$, Liang $H, F M$, Lifang $H, N W, X Z, L Z, X C, Z M$, Manuscript drafting: Jianfeng Z, LC, GW, YC. Critical revision of the manuscript for important intellectual contents: Jianfeng Z. Statistical analysis: LC, XL, JT, YC. Financial support: Jianfeng $Z$ and WW. Administrative and technical support: Jianfeng Z, ZS, ZL, Jianping Z, DW, GH, WW. Supervision: Jianfeng Z, WW.

\section{ADDITIONAL INFORMATION}

The online version of this article (https://doi.org/10.1038/s41423-020-0492-x) contains supplementary material.

Competing interests: The authors declare no competing interests.

\section{REFERENCES}

1. Mahase, E. Covid-19: WHO declares pandemic because of "alarming levels" of spread, severity, and inaction. BMJ 368, m1036 (2020).

2. Du, Y. et al. Clinical features of 85 fatal cases of COVID-19 from Wuhan. A retrospective observational study. Am. J. Respiratory Crit. Care Med. 201, 1372-1379 (2020).

3. Cao, Y., Liu, X., Xiong, L. \& Cai, K. Imaging and clinical features of patients with 2019 novel coronavirus SARS-CoV-2: a systematic review and meta-analysis. $J$. Med. Virol. https://doi.org/10.1002/jmv.25822 (2020).

4. Liang, W. et al. Development and validation of a clinical risk score to predict the occurrence of critical illness in hospitalized patients with COVID-19. JAMA Intern. Med. e202033. https://doi.org/10.1001/jamainternmed.2020.2033 (2020).

5. Vénéreau, E., Ceriotti, C. \& Bianchi, M. E. DAMPs from cell death to new life. Front. Immunol. 6, 422 (2015).

6. Krysko, D. V. et al. Immunogenic cell death and DAMPs in cancer therapy. Nat. Rev. Cancer 12, 860-875 (2012).

7. Chan, J. K. et al. Alarmins: awaiting a clinical response. J. Clin. Investig. 122, 2711-2719 (2012).

8. Rudd, K. E. et al. Association of the Quick Sequential (Sepsis-Related) Organ Failure Assessment (qSOFA) Score With Excess Hospital Mortality in Adults With Suspected Infection in Low- and Middle-Income Countries. Jama. 319, 2202-2211 (2018).

9. Entezari, M. et al. Inhibition of extracellular HMGB1 attenuates hyperoxia-induced inflammatory acute lung injury. Redox Biol. 2, 314-322 (2014).

10. Huebener, P. et al. The HMGB1/RAGE axis triggers neutrophil-mediated injury amplification following necrosis. J. Clin. Investig. 125, 539-550 (2015).

Open Access This article is licensed under a Creative Commons Attribution 4.0 International License, which permits use, sharing, adaptation, distribution and reproduction in any medium or format, as long as you give appropriate credit to the original author(s) and the source, provide a link to the Creative Commons license, and indicate if changes were made. The images or other third party material in this article are included in the article's Creative Commons license, unless indicated otherwise in a credit line to the material. If material is not included in the article's Creative Commons license and your intended use is not permitted by statutory regulation or exceeds the permitted use, you will need to obtain permission directly from the copyright holder. To view a copy of this license, visit http://creativecommons. org/licenses/by/4.0/.

(c) The Author(s) 2020 